\title{
A cloud provider selection scheme for cloud storage services
}

\author{
Ryoma Kaneko, Praphan Pavarangkoon, and Eiji Oki ${ }^{\text {a) }}$ \\ Department of Communication Engineering and Informatics, The University of \\ Electro-Communications, 1-5-1 Chofugaoka, Chofu, Tokyo 182-8585, Japan
}

a)eiji.oki@uec.ac.jp

\begin{abstract}
This paper proposes a cloud provider selection scheme for cloud storage services to minimize the total cost of usage. The proposed scheme satisfies the user's requirements using multiple cloud providers. The proposed scheme considers the time-dependent unavailability and multiple-user environment. The cloud provider selection problem is formulated as a linear programming problem. We evaluate the total cost of usage of our scheme for the various allowable numbers of cloud providers. Numerical results show that the proposed scheme with multiple cloud providers reduces the total cost of usage, compared to that with only one cloud provider. The total cost of usage is more reduced if the time-dependent scheme is used.
\end{abstract}

Keywords: cloud network, cost model, optimization, linear programming Classification: Network

\section{References}

[1] S. Chaisiri, B.-S. Lee, and D. Niyato, "Optimal virtual machine placement across multiple cloud providers," IEEE Asia-Pacific Services Computing Conference, 2009. APSCC 2009, Singapore, pp. 103-110, 2009. DOI:10. 1109/APSCC.2009.5394134

[2] I. Houidi, M. Mechtri, W. Louati, and D. Zeghlache, "Cloud service delivery across multiple cloud platforms," 2011 IEEE International Conference on Services Computing (SCC), Washington, DC, pp. 741-742, 2011. DOI:10.1109/ SCC.2011.107

[3] "CloudSquare Service Status," Mar. 2016. [Online]. Available: https:// cloudharmony.com/status

[4] "CloudSquare Provider Directory," Mar. 2016. [Online]. Available: https:// cloudharmony.com/cloudsquare

[5] "Best Cloud Storage Providers of 2015," Mar. 2016. [Online]. Available: http:// www.cloudwards.net/comparison/

[6] J. Chase, R. Kaewpuang, W. Yonggang, and D. Niyato, "Joint virtual machine and bandwidth allocation in software defined network (SDN) and cloud computing environments," 2014 IEEE International Conference on Communications (ICC), pp. 2969-2974, Jun. 2014. DOI:10.1109/ICC.2014.6883776

[7] R. Kaneko, P. Pavarangkoon, and E. Oki, "Virtual machine selection scheme considering reliability for cloud services," 21st Asia-Pacific Conference on Communications (APCC 2015), Kyoto, Japan, 2015. DOI:10.1109/APCC.2015. 7412546 


\section{Introduction}

A cloud storage service is used to store data of users over the Internet. A user uploads his/her own data to a storage served by a cloud provider, and accesses the data from anywhere on any devices connected to the Internet. The cloud storage service is sometimes not available due to its maintenance or failure.

Several works presented schemes related to multiple cloud providers [1, 2]. The work in [1] presented a cloud service selection scheme considering optimal virtual machine placement. The work in [2] presented a scheme to optimize allocation of nodes and links so as to minimize the total cost of usage.

Each cloud service consists of various parameters. Unavailability referring to the probability that the cloud service is not available. A relationship between cost of usage and unavailability exists. Several websites collect and announce information about the status and the prices of cloud providers $[3,4,5]$. The works in $[6,7]$ considered availability of cloud providers to select virtual machines in cloud services. The unavailability of a cloud provider is time-dependent. The unavailable time depends on maintenance schedule. The user's requirement is time-dependent. The requirement of unavailability of a cloud provider depends on working time of users. Moreover, to support multiple users, the broker satisfies the users' requirements, e.g., unavailability, total cost, and transfer rate.

To satisfy user's requirement of unavailability, the conventional cloud provider selection scheme [6] considers only one cloud provider with low unavailability. Generally, the usage cost of cloud provider with low unavailability is higher than that with high unavailability. However, it is able to reduce the total cost when the combination of cloud providers with high unavailability.

This paper proposes a cloud provider selection scheme for cloud storage services to minimize the total cost of usage. The cloud provider selection scheme is used by the broker and selects multiple cloud providers with considering the time-dependent unavailability and users' transfer-rate requirement in multiple-user environment. The proposed scheme allows users to select multiple cloud providers, while minimizing the total cost of usage. We evaluate the performance of the proposed scheme by varying the number of allowable cloud providers. The results show that the proposed scheme with multiple cloud providers reduces the total cost of usage, compared to that with single cloud provider.

\section{Mathematical model}

The objective of the cloud provider selection problem is to minimize the total cost of usage for a broker. We formulate this problem considering the combination of the cloud providers to satisfy the users' requirements as a linear programming problem.

Let $i \in T$ be a cloud provider, where $T$ denotes a set of cloud providers. Let $u \in R$ be a user, where $R$ is a set of users. Let $t \in J$ be a time, where $J$ is a set of times. We denote $|T|=N,|R|=M$, and $|J|=L$.

The proposed scheme selects multiple cloud providers. $k_{u}$ is the allowable number of cloud providers for user $u$. Cloud provider $i$ charges user $u$ according to bandwidth usage. $d_{i}$ denotes the cost of bandwidth usage between a user and cloud 
provider $i . c_{i}$ denotes the usage cost of cloud provider $i$. Therefore, the total cost is the summation of usage cost of cloud provider $c_{i}$ and cost of bandwidth usage $d_{i}$. $x_{u i}$ is a binary decision variable that is set to one if cloud provider $i$ provides a service for user $u$ and zero otherwise.

We consider the time-dependent unavailability. Each user has the requirements for the unavailability of the cloud services at each time. $U_{r e q}^{u}(t)$ denotes the acceptable unavailability of user $u$ at time $t$.

$U_{i}(t)$ denotes the unavailability of cloud provider $i$ at time $t$. Therefore, to satisfy the user's requirement of unavailability, the unavailability of the selected cloud provider, $U_{i}(t)$, must be equal to or greater than the user's acceptable unavailability, $U_{r e q}^{u}(t)$. For non-time-dependent unavailability, $U_{r e q}^{u}$ and $U_{i}$ do not depend on time. In addition, the user sets only $U_{r e q}^{u}$, which is the strictest unavailability of the day that the user requires.

For a multiple-user environment, users use specific cloud providers at the same time, it could affect the performance of the cloud provider (e.g., transfer rate). Suppose that user $u$ has the requirement for the transfer rate $S_{\text {req }}^{u}$. The remaining bandwidth is obtained by deducting all the transfer rates of the users of the cloud provider $i$ from bandwidth $S_{i}$. A user is able to use all the remaining bandwidth if it is equal to or greater than $S_{r e q}^{u}$.

To satisfy the user's acceptable unavailability, the unavailabilities of the selected cloud providers is formulated, as shown in Eq. (1).

$$
\prod_{i \in T: x_{u i}=1} U_{i}(t) \leq U_{r e q}^{u}(t), \forall u \in R, \forall t \in J
$$

The left side of Eq. (1) expresses the unavailability that all the selected cloud providers are unavailable.

Objective

$$
\min \sum_{u \in R} \sum_{i \in T} x_{u i}\left(c_{i}+d_{i}\right)
$$

Constraints

$$
\begin{gathered}
\sum_{i \in T} x_{u i} \log U_{i}(t) \leq \log U_{r e q}^{u}(t), \forall u \in R, \forall t \in J \\
\sum_{i \in T} x_{u i} \leq k_{u}, \forall u \in R \\
S_{i}-\sum_{u \in R: u \neq u^{\prime}} x_{u i} S_{r e q}^{u} \geq S_{r e q}^{u^{\prime}}, \forall i \in T, \forall u^{\prime} \in R \\
x_{u i} \in\{0,1\}, \forall i \in T, \forall u \in R
\end{gathered}
$$

The objective function in Eq. (2a) determines the combination of cloud providers so as to minimize the total cost of usage. Equation $(2 b)$ is transformed from Eq. (1) to a linear form by taking the logarithmic of both sides. This equation ensures that the probability that all the selected cloud providers are unavailable does not exceed the user's acceptable unavailability. Moreover, it ensures that the user selects at least one cloud provider. Equation (2c) expresses that the number of selected cloud providers does not exceed the number of allowable cloud providers $k_{u}$ for user $u \in R$. Equation (2d) ensures that the cloud provider's remaining transfer rate is equal to or more than the user's acceptable transfer-rate requirements $S_{r e q}^{u}$. 


\section{Performance evaluation}

We evaluate the performance of the proposed scheme. First, the total cost of usage of the proposed scheme is investigated with the various allowable numbers of cloud providers $k_{u}$ on both non-time-dependent and time-dependent scenarios. We set $N=10, M=1$, and $L=3$. We assume that one user requests cloud storage services to a broker. The broker gets the minimum total cost of usage among multiple cloud providers. The user has different requirements for the three hours in, EarlyMorning (2AM-8AM), Daytime (8AM-4PM), Night (4PM-2AM), where $t=1,2,3$ are EarlyMorning, Daytime, and Night, respectively. All $d_{i}$ is set to 1 . We set $U_{r e q}^{1}(1)=0.083, U_{r e q}^{1}(2)=0.09$, and $U_{r e q}^{1}(3)=0.000008$. In the non-timedependent scenario, we set $U_{\text {req }}=0.000008$. In order to investigate the effect of time-dependent unavailability, we set $S_{i}=10,000,000$ and $S_{r e q}^{u}=10$. With $S_{i} \gg S_{r e q}^{u}$, Eq. (2d) does not affect any cloud provider section.

Second, the effect of bandwidth limitation is investigated. We set $M=5$. All the user's acceptable unavailability $U_{\text {req }}$ is set to 0.000008 . For bandwidth limitation, $S_{\text {req }}^{u}=5$ is set for all $u$, and the transfer rates of each cloud provider $S_{i}$ is set to $10,15,20,25,30,35,38,43,48$, and 50 , respectively. The other parameters are the same with those of the non-time-dependent scenario above.

Table I. Parameter settings

\begin{tabular}{crrrrr}
\hline & & Non-time-dependent & \multicolumn{3}{c}{ Time-dependent } \\
\cline { 3 - 6 } Cloud provider $i$ & $c_{i}$ & $U_{i}$ & $U_{i}(1)$ & $U_{i}(2)$ & $U_{i}(3)$ \\
\hline 1 & 4 & 0.014 & 0.009 & 0.012 & 0.014 \\
2 & 5 & 0.009 & 0.009 & 0.009 & 0.009 \\
3 & 6 & 0.022 & 0.022 & 0.005 & 0.006 \\
4 & 13 & 0.005 & 0.005 & 0.005 & 0.005 \\
5 & 14 & 0.025 & 0.025 & 0.016 & 0.018 \\
6 & 15 & 0.016 & 0.016 & 0.016 & 0.016 \\
7 & 20 & 0.001 & 0.0009 & 0.0008 & 0.001 \\
8 & 30 & 0.0008 & 0.0008 & 0.0008 & 0.0008 \\
9 & 50 & 0.0005 & 0.0004 & 0.0005 & 0.000008 \\
10 & 70 & 0.000008 & 0.000008 & 0.000008 & 0.000008 \\
\hline
\end{tabular}

Table I shows the parameter settings of cloud providers to study the basic characteristics of the proposed scheme in comparison of the non-time-dependent and time-dependent scenarios. A cloud provider with an even index number provides the minimum unavailability of the day that is provided by the cloud provider with the previous odd index number.

Fig. 1 shows the total cost of usage dependent on the maximum allowable number of cloud providers, $k_{u}$, for the non-time-dependent and time-dependent scenarios. The total cost of usage is normalized by that of the non-time-dependent and time-dependent scenario with $k_{u}=1$. The proposed scheme allows to select the cloud providers with lower total cost of usage while satisfying the requirement 


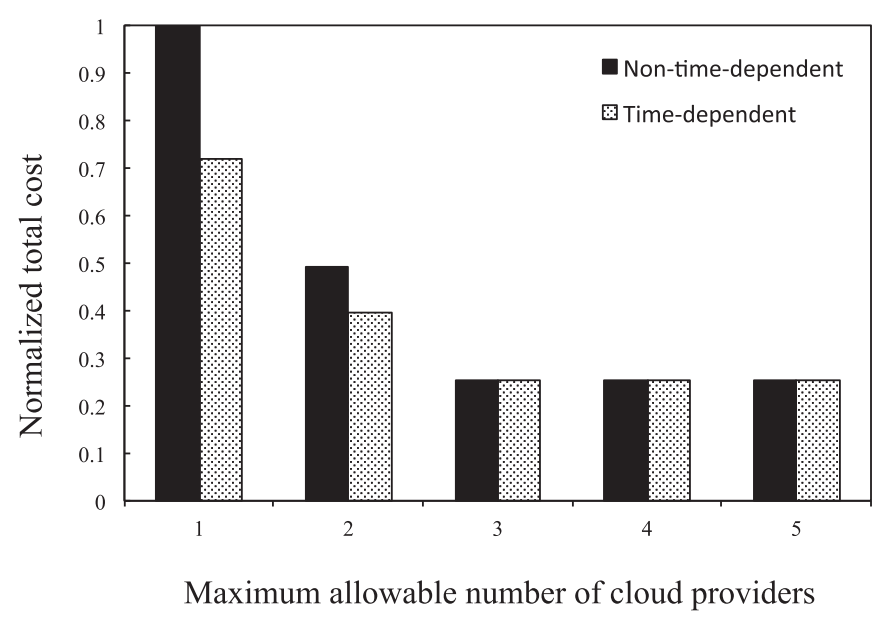

Fig. 1. Total cost of usage dependent on the maximum allowable number of cloud providers for non-time-dependent and timedependent scenarios.

of unavailability. The proposed scheme reduces the total cost of usage as the maximum allowable number of cloud providers increases. However, the total costs of usage on both scenarios are not improved after the maximum allowable number of cloud provider exceeds 3 , which we call a saturated point. The saturated point becomes small as $d_{i}$ increases. This is because that using more cloud providers increases the total bandwidth cost, which is considered as a penalty. This effect becomes more significant when $d_{i}$ increases.

The total cost of time-dependent scenario is lower than that of the non-timedependent. The time-dependent scenario allows the user to require the minimum requirements for unavailability at each time. The user selects cloud providers with acceptable cost while the users do not require low unavailability. The usage cost of cloud providers $c_{i}$ selected by the time-dependent scenario is less than that of nontime-dependent scenario. For this reason, the total cost of time-dependent scenario is less than that selected by the non-time-dependent scenario.

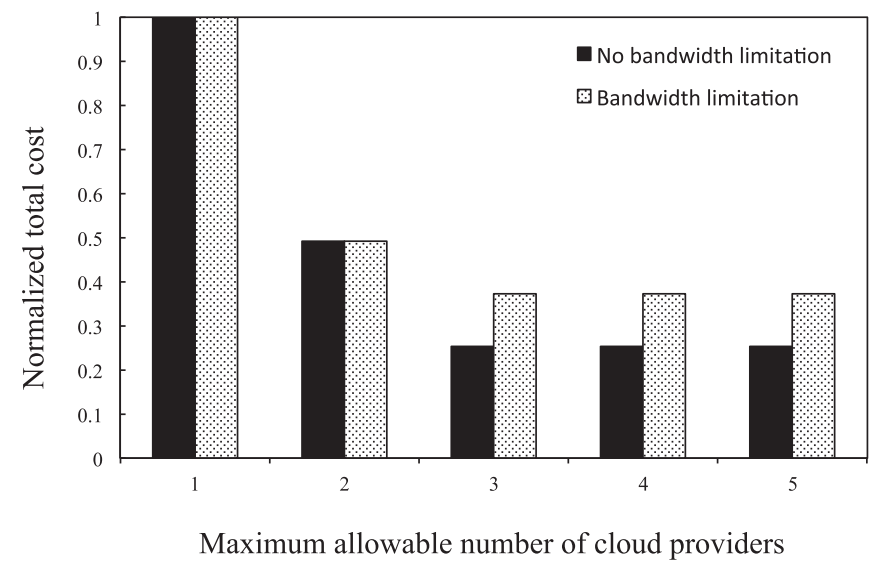

Fig. 2. Total cost of usage in comparison of non-bandwidth limitation and bandwidth limitation. 
bandwidth is narrow or the user uses high bandwidth, Eq. (2d) is effective to avoid the situation that the user uses more bandwidth than cloud provider's bandwidth. The bandwidth limitation allows a multiple-user environment to satisfy their requirements of bandwidths.

\section{Conclusions}

This paper proposed a cloud provider selection scheme for cloud storage services to minimize the total cost of usage. The proposed scheme considers time-dependent unavailability, multiple-user environment, and bandwidth limitation. The problem of cloud provider selection is formulated as a linear programming problem. The results showed that the proposed scheme considering multiple cloud providers reduces the total cost of usage, compared to that considering only one cloud provider. 